\title{
The relationship between the PD-I/PD-LI pathway and DNA mismatch repair in cervical cancer and its clinical significance
}

This article was published in the following Dove Press journal:

Cancer Management and Research

\author{
Yang-chun Feng ${ }^{1,2}$ \\ Wen-li Ji ${ }^{3}$ \\ $\mathrm{Na} \mathrm{Yue}{ }^{3}$ \\ Yan-chun Huang ${ }^{2}$ \\ Xiu-min $\mathrm{Ma}^{\prime}$
}

'Clinical Laboratory Center, The First Affiliated Hospital of Xinjiang Medical University, ${ }^{2}$ Clinical Laboratory Center, ${ }^{3}$ Clinical Pathology Center, Tumor Hospital Affiliated to Xinjiang Medical University, Urumqi, People's Republic of China
Correspondence: Yan-chun Huang Clinical Laboratory Center, Tumor Hospital Affiliated to Xinjiang Medical University, No 789 Suzhou Road, Urumqi 8300 I I, People's Republic of China Email xjhyc668899@sina.com

Xiu-min Ma

Clinical Laboratory Center, The First Affiliated Hospital of Xinjiang Medical University, No 137 Liyushan Road, Urumqi 830054, People's Republic of China

Email mxm20160722@sina.com
Background: According to recent clinical observations, deficient DNA mismatch repair (dMMR) is capable of improving antitumor effects of the PD-1/PD-L1 pathway, suggesting that dMMR may act as a prognostic indicator of PD-1/PD-L1 antibody drugs. In this study, we examined the dMMR and PD-1/PD-L1 expression, as well as explored the correlation of dMMR status with PD-1/PD-L1 expression in cervical cancer patients, in order to optimize cervical cancer patient selection for PD-1/PD-L1 antibody drug treatment, which is helpful to avoid adverse effects and keep costs manageable.

Methods: Sixty-six tissue samples from patients with squamous cell carcinoma were collected, and data of their clinical characteristics were also gathered. Based on these samples, the expression levels of MLH1, MSH2, and PD-L1 in cancer cells were tested by immunohistochemical assay (IHC). Moreover, PD-1/PD-L1 expression in tumor-invading lymphocytes (TILs) was detected by IHC as well. Six single-nucleotide-repeat markers of microsatellite instability (MSI), including NR-27, MONO-27, BAT-25, NR-24, NR-21, and BAT-26, were tested by capillary electrophoresis sequencer analysis. According to expression of MLH1, MSH2 and the MSI test, all 66 cases were divided into dMMR or proficient DNA mismatch repair (pMMR) groups. The comparisons of dMMR and PD-L1 in cancer cells and of PD-1/PD-L1 in TILs were conducted categorized by age, childbearing history, history of abortion, ethnicity, and cancer cell differentiation subgroup. Furthermore, PD-L1 levels in cancer cells and PD-1/PD-L1 in TILs were analyzed and compared in both dMMR and pMMR subgroups.

Results: Of the patient samples, 25.8\% were associated with dMMR. PD-L1 in cancer cells, PD-L1 in TILs, and PD-1 in TILs took up 59.1\%, 47.0\%, and 60.6\%, respectively. The data indicated that both $\mathrm{dMMR}$ and PD-L1 overexpression resulted from lower cancer differentiation, more incidences of childbearing, and a history of abortion. Abortion could significantly increase PD-1 expression levels in TILs. Additionally, more incidence of childbearing or older age (35-55 years) was able to upregulate PD-L1 expression in TILs. Statistical difference of PD-L1 in cancer cells could be observed between dMMR and pMMR subgroups. In the dMMR group, PD-L1 in cancer cells and PD-1 in TILs had no correlation $(r=0.161, p=0.537)$, but in the pMMR group, they had good correlation $\left(r_{\mathrm{s}}=0.645, p<0.001\right)$.

Conclusion: According to prior studies and our own experiments, PD-L1 in both cancer cells and TILs and PD-1 in TILs are widely observed in cervical cancer patients, indicating that there may be potential to apply PD-1/PD-L1 antibody drugs in cervical cancer. dMMR patients are associated with higher PD-L1 expression compared with pMMR ones, which suggested that PD-1/PD-L1 antibody drugs may work well in dMMR cervical cancer patients. Moreover, in patients with more incidences of childbearing or abortion, dMMR may be a molecular detection target for clinical application of PD-1/PD-L1 antibody drugs.

Keywords: programmed cell death 1, programmed cell death 1 ligand 1, DNA mismatch repair system, cervical cancer 


\section{Introduction}

PD-1/PD-L1, including PD-1 in lymphocyte and PD-L1 in tumor cell, dendritic lymphocyte, and placenta cell, etc, also called the tumor immune checkpoint, plays critical roles in human immune regulation pathways. PD1/PD-L1 represents an adaptive immune resistance mechanism by providing inhibitory signals, such as T cell inactivation and inhibition. ${ }^{1}$

The DNA mismatch repair (MMR) system is coded by MMR genes and has a series of specific proteins. This system is able to keep DNA replication fidelity by maintaining genetic integrity and stability, and avoiding replication mutations. MMR consists of six core proteins, such as MSH2, MLH1, MSH3, MSH6, PMSH1, and PMSH2. Among these, MLH1 and MSH2 play leading and dominant roles. ${ }^{2,3}$ Deficient MMR (dMMR) is the status when MMR proteins are deficient or biologically malfunction. Another MMR subgroup is proficient MMR (pMMR), a counterpart phenomenon in which the patient has normal and biological function MMR expression. In the clinic, MMR proteins were tested by immunohistochemistry assay (IHC). The biological functions of MMR, including microsatellite instability (MSI) and microsatellite stability (MSS), were analyzed by polymerase chain reaction (PCR).

Prior studies identified that dMMR is able to improve the antitumor efficacy of PD-1/PD-L1 antibody drugs, which suggested that $\mathrm{dMMR}$ may be the first prognostic indicator of PD-1/PD-L1 antibody drugs. ${ }^{4-7}$ PD-1/PD-L1 antibody drugs have already exhibited efficacy in treating melanoma, non-small cell lung cancer, and colorectal cancer, etc. Immunotherapy options like PD-1/PD-L1 antibody drugs are starting to be explored in cervical cancer, and therefore PD-1/ PD-L1 antibody drugs are regarded as a potential strategy for cervical cancer patients. ${ }^{8}$ Due to MMR being implicated in the PD-1/PD-L1 regulations, we thus hypothesize that MMR may have clinical value to regulate PD-1/PD-L1 pathways in cervical cancer. In this study, to optimize patient selection for PD-1/PD-L1 antibody drugs, we assessed the association between PD-1/PD-L1 and MMR in cervical cancer. ${ }^{9}$

\section{Materials and methods}

\section{Sample source}

Sixty-six tissue samples of squamous cell carcinoma patients from the Tumor Hospital Affiliated to Xinjiang Medical University were collected, among them 36 were Uygur women and 30 were Han women. Along with the tumor paraffin specimens, clinical characteristics were also collected. Every paraffin specimen contained five pathological tissue sections. Meanwhile, normal tissue and tumor tissue samples for PCR were also collected. The Ethics Committee of The Tumor
Hospital Affiliated to Xinjiang Medical University approved the study and the consent procedure. Written informed consent was obtained from all subjects before the study.

\section{Immunohistochemistry}

\section{Experimental operation}

Monoclonal antibodies of MLH1, MSH2, PD-L1, and PD-1 were bought from Golden Bridge Biotechnology Co. Ltd (Beijing, People's Republic of China). H\&E staining was used to assess the pathological slices.

The detection method was IHC streptavidin-perosidase and diaminobenzidine chromogenic detection. All slices were produced using the following procedures: dewaxing, rehydration, washing in xylene, ethanol, steamed classification, boiling water to wash, dissolving endogenous peroxide physical activity by incubation with $3 \% \mathrm{H}_{2} \mathrm{O}_{2}$, antigen repair, and dyeing. Phosphate-buffered saline (PBS), rather than the first antibody, was the negative control; the previous positive specimen was the positive control.

\section{Result criterion}

MLH1 and MSH2 proteins stained in the nucleus. We selected 10 horizon at high magnification and calculated the proportion of positive cells; positive cell criterion is in the nucleus appear tan particles and loss of nucleus staining means lacking expression of the proteins. For PD-L1 expression in cancer cells, we chose the nests of cervical cancer including more than 100 cancer cells and then calculated the proportion of positive cells ( $10 \%$ or less was negative, between $10 \%$ and $50 \%$ was positive, more than $50 \%$ was strong positive); positive criterion is in the cytomembrane and cytoplasm or both at the same time appeared tan particles. For PD-L1 and PD-1 protein expression in tumor-invading lymphocytes (TILs), we selected 10 horizon at high magnification and calculated the proportion of positive cells $(10 \%$ or less was negative, more than $10 \%$ was positive); positive criterion is in the cytomembrane and cytoplasm or both at the same time appeared tan particles. Expression of MLH1 and MSH2 in cancer cells is shown in Figure 1(A-D), PD-L1 in cervical cancer cells in Figure 1(E-F), PD-1 in TILs in Figure 1(G-H), and PD-L1 in TILs in Figure 1(I-J).

\section{MSI test}

\section{Experiment operation}

1) Normal tissue and tumor tissue samples from 66 patients were collected, and DNA from every sample was extracted; 2) the predefined nucleotide site was amplified by multiple fluorescent PCR, and six single-nucleotide-repeat markers 


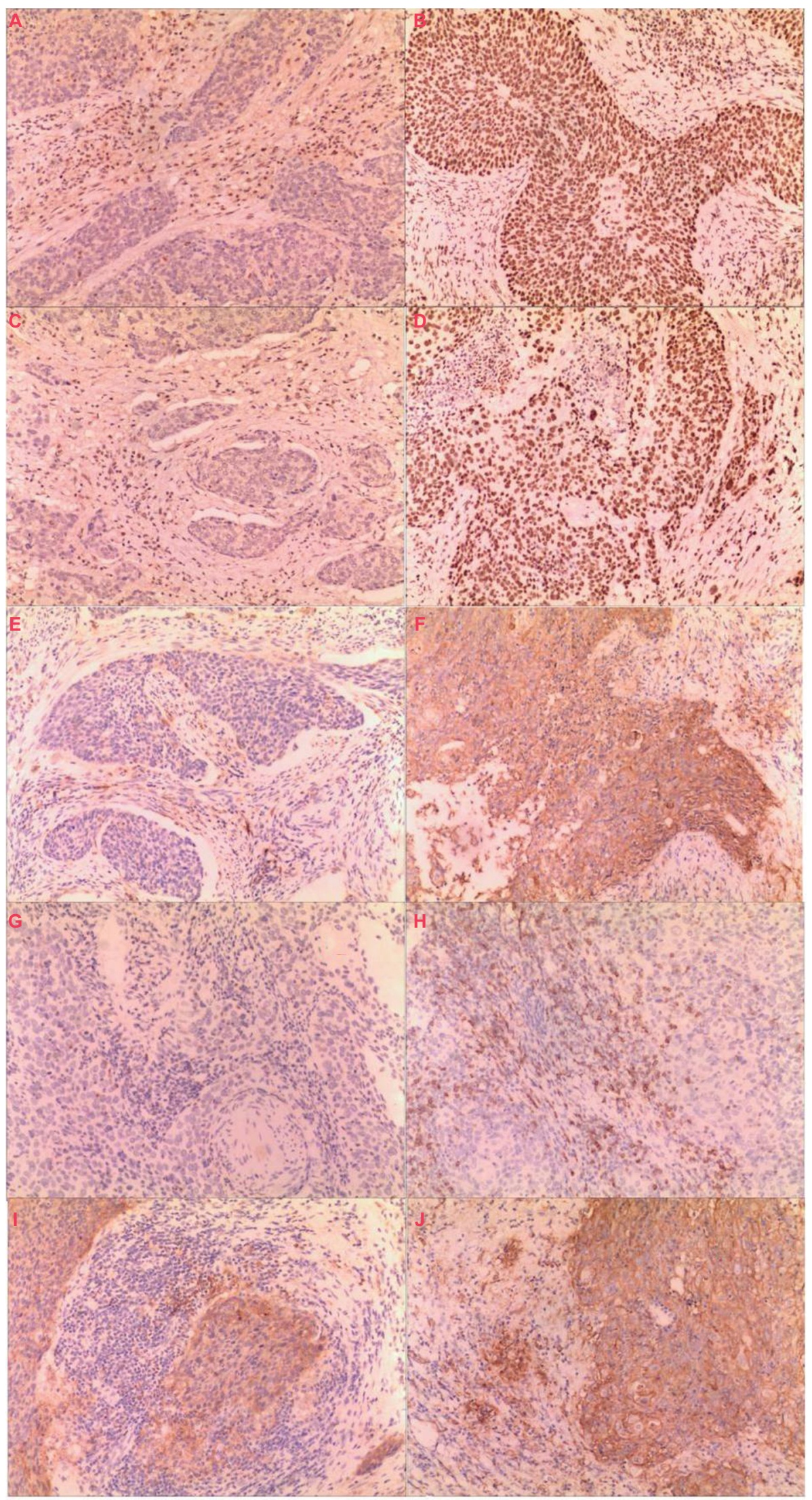

Figure I Expression of MLHI, MSH2, PD-LI, and PD-I (I00× amplification).

Notes: Negative (A) and positive (B) expression of MLHI in cervical cancer cells. Negative (C) and positive (D) expression of MSH2 in cervical cancer cells. Negative (E) and positive (F) expression of PD-LI in cervical cancer cells. Negative (G) and positive (H) expression of PD-I in TILs. Negative (I) and positive (J) expression of PD-LI in TILs. Abbreviation: TIL, tumor-invading lymphocyte. 
of the nucleotide site, including NR-27, MONO-27, BAT25, NR-24, NR-21, and BAT-26, were tested by MSI. Two five-nucleotide-repeat markers (Penta C and Penta D) and one Amelo marker (Amelo) were used to identify potential sample mixing or potential pollution. Detailed information of the MSI test by PCR is presented in Table 1;3) the nucleotide sequence was examined and analyzed by capillary electrophoresis sequencer.

\section{Result criterion}

MSS showed no change in any single-nucleotide-repeat markers. MSI showed a change existed in one or more singlenucleotide-repeat markers. Examples of the MSS and MSI results are shown in Figures 2 and 3.

\section{Statistical analysis}

Data presented in this study were analyzed by SPSS 17.0 statistical software. Comparison of multiple sample rates and correlation analysis between two classification variables were measured by chi-square correlation analysis. The inspection level was $\alpha=0.05$, and $p<0.05$ indicated statistical difference.

\section{Results}

\section{MMR classifications of 66 cases}

In 66 patients, 49 cases (74.2\%) were both MSH2 and MLH1 positive, either MSH2 or MLH1was lacking in 15 cases (22.7\%), and both MSH2 and MLH1 were lost in only two cases $(3.1 \%)$. Four patients $(6.1 \%)$ were identified as MSI, who also had MSH2 or/and MLH1 locking expression. Lacking expression of either or both MLH1 and MSH2 (including MSI) was classified into dMMR. In total, the dMMR group included 17 cases and the pMMR group contained 49 cases.

\section{PD-LI in cancer cells, rather than PD-I and PD-LI in TILs, has statistical difference between the two MMR groups}

Strong positive, positive, and negative PD-L1 expression in cervical cancer cells was present in 21 cases (31.8\%), 18 cases $(27.3 \%)$, and 27 cases $(40.9 \%)$, respectively. The comparison of PD-L1 expression in cancer cells between dMMR and pMMR groups is shown in Table 2, revealing that PD-L1 expression had statistical difference between the two MMR groups. Furthermore, 31 of all 66 cases (47.0\%) were associated with positive PD-1 in TILs. Forty out of 66 were implicated with positive TILs PD-L1, making up 60.6\% of all cases. The results showed that both PD-1 and PD-L1

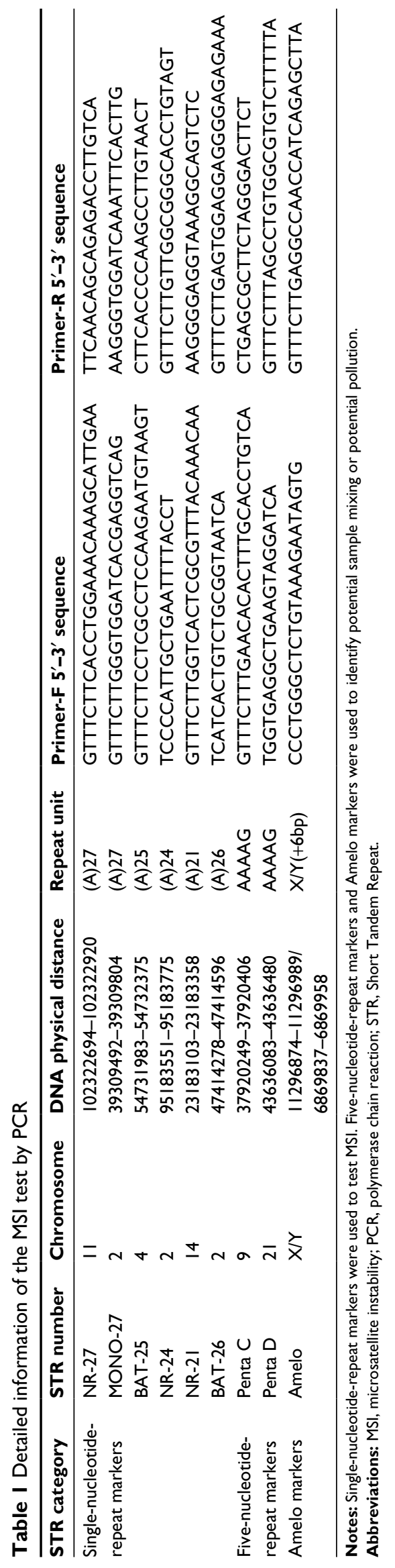




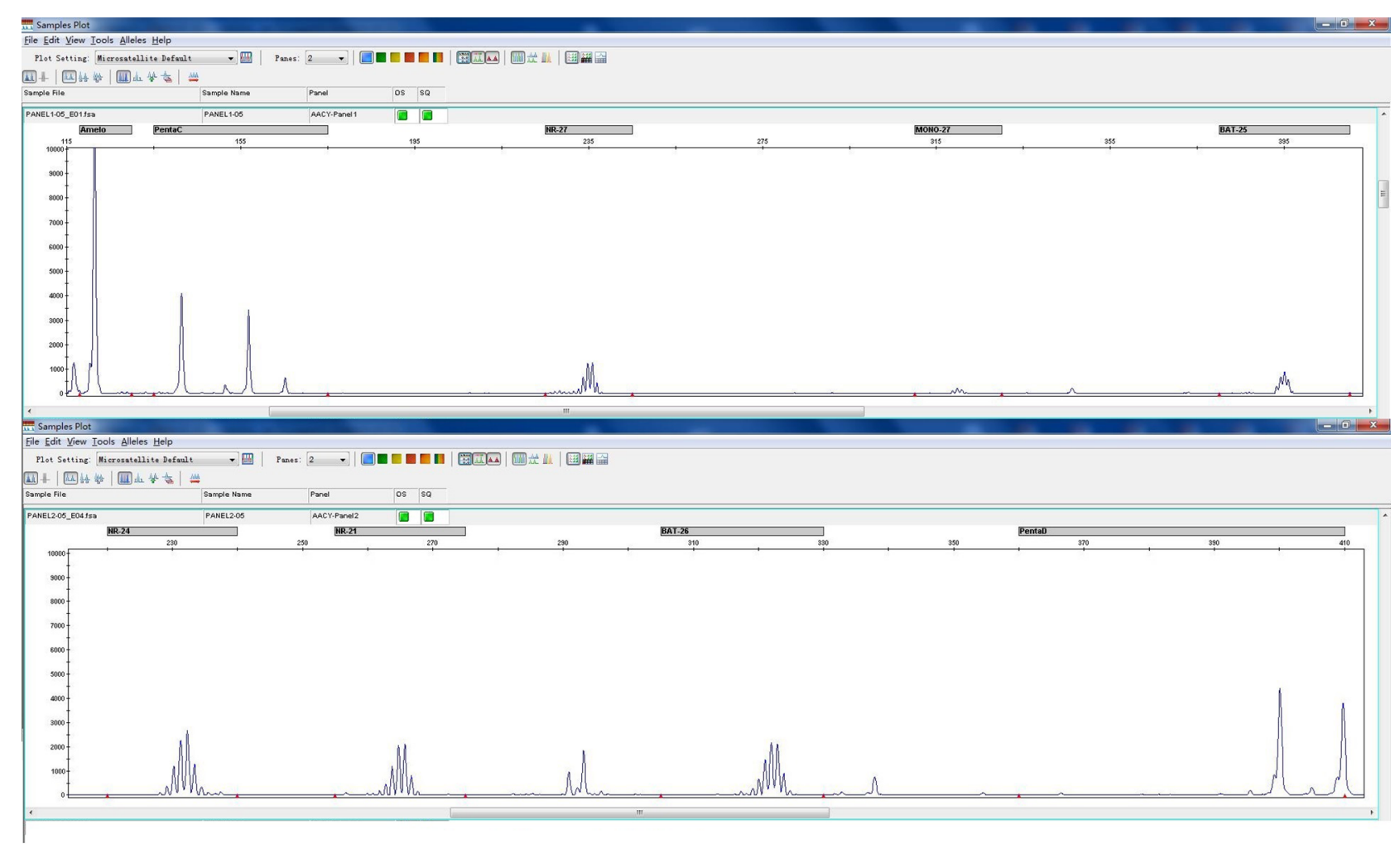

Figure 2 Example of microsatellite stability status.

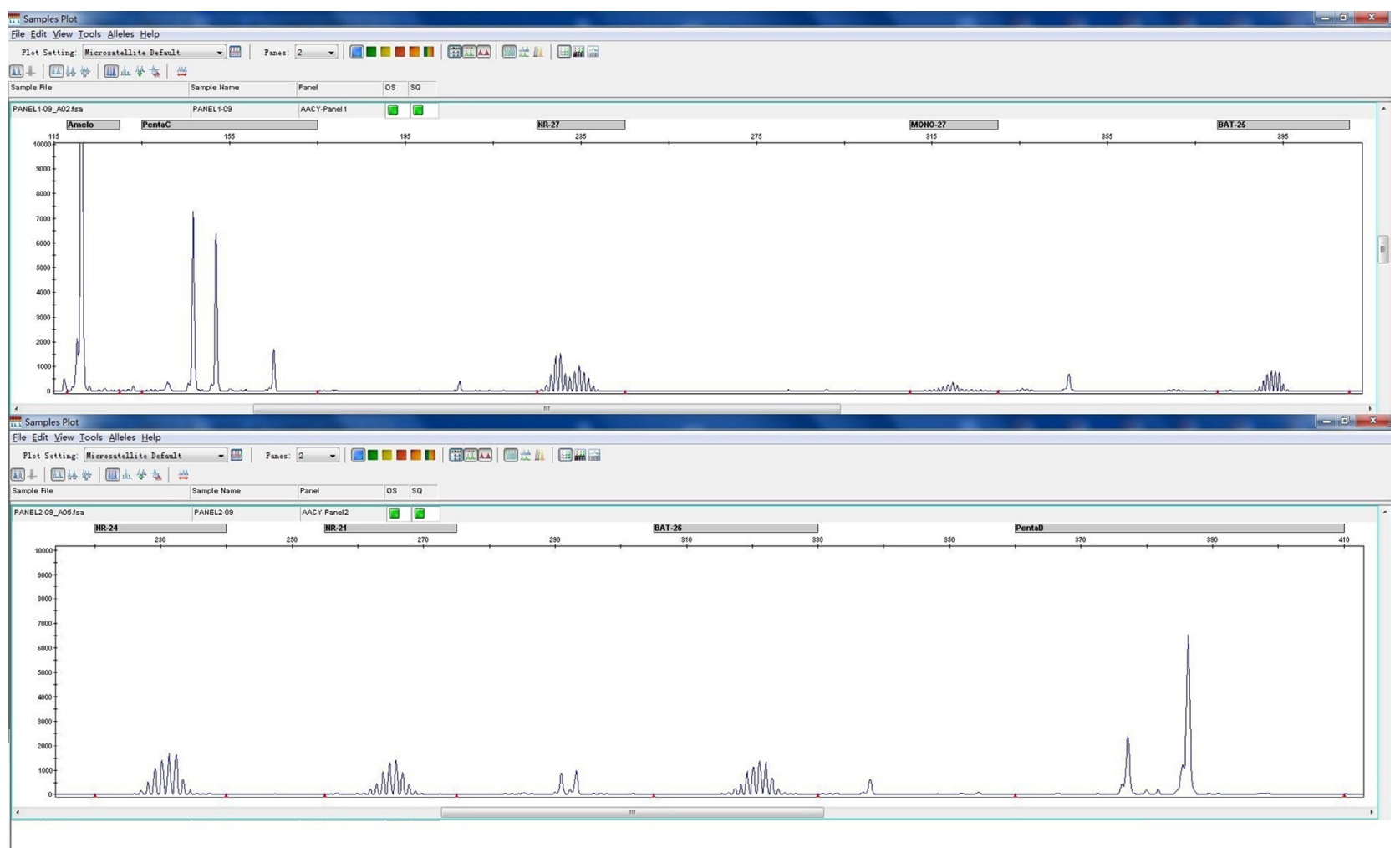

Figure 3 Example of microsatellite instability status (MONO-27 and NR-27 had changes). 
expression in TILs had no statistical difference between the two MMR groups (Table 3).

\section{The relationship between PD-I and PD- $\mathrm{LI}$ in different MMR groups}

In the dMMR group, PD-L1 in cancer cells and PD-1 in TILs had no correlation $\left(r_{\mathrm{s}}=0.161, p=0.537\right)$ and PD-L1 in cancer cells and PD-L1 in TILs had no correlation $\left(r_{\mathrm{s}}=0.250\right.$, $p=0.334)$. However, PD-L1 and PD-1 in TILs exhibited strong correlation $\left(r_{\mathrm{s}}=0.509, p=0.037\right)$. In the pMMR group, there was good correlation between PD-L1 in cancer cells and PD-1 in TILs $(r=0.645, p<0.001)$, and between PD-L1 in TILs and PD-1 in TILs $(r=0.439, p=0.002)$. The correlation between PD-L1 in cancer cells and PD-L1 was $r_{\mathrm{s}}=0.507, p<0.001$.

\section{Clinical significance of MMR and the PD-I/PD-LI pathway in 66 patients}

Clinical data of 66 patients were then categorized by age, childbearing history, abortion history, ethnicity, and cancer cell differentiation (Table 4). Comparisons of MMR and PD-L1 in cancer cells and PD-1/PD-L1 in TILs in the different clinical subgroups were performed (Figure 4). The results showed that lower cancer differentiation and more incidences of childbearing and abortion predicted dMMR status. Moreover, more incidences of childbearing and abortion were association with PD-L1 expression levels in cancer cells. Additionally, abortion could enhance PD-1 expression in TILs, while more incidences of childbearing history and older age (35-55 years) increased PD-L1 expression in TILs.

Table 2 Comparison of cancer cells PD-LI expression in different MMR groups

\begin{tabular}{lllll}
\hline MMR group & N & \multicolumn{2}{l}{ PD-LI expression* } \\
\cline { 3 - 5 } & & Negative & Positive & Strong positive \\
\hline dMMR & 17 & $2(11.8 \%)$ & $4(23.5 \%)$ & $11(64.7 \%)$ \\
PMMR & 49 & $25(51.0 \%)$ & $14(28.6 \%)$ & $10(20.4 \%)$ \\
\hline
\end{tabular}

Note: ${ }^{*} \chi^{2}=12.656, p=0.002$.

Abbreviations: IMMR, deficient DNA mismatch repair; MMR, DNA mismatch repair; PMMR, proficient DNA mismatch repair.

Table 3 Comparison of TIL PD-I and PD-LI expression in different MMR groups

\begin{tabular}{lllllll}
\hline MMR & \multirow{2}{*}{ N } & \multicolumn{2}{l}{ PD-I expression* } & & \multicolumn{2}{l}{ PD-LI expression** } \\
\cline { 3 - 4 } \cline { 6 - 7 } & & Negative & Positive & & Negative & Positive \\
\hline dMMR & 17 & $8(47.1 \%)$ & $9(52.9 \%)$ & & $6(42.9 \%)$ & II $(57.1 \%)$ \\
PMMR & 49 & $29(57.8 \%)$ & $20(42.2 \%)$ & & $20(57.8 \%)$ & $29(42.2 \%)$ \\
\hline
\end{tabular}

Notes: $\chi^{2}=0.753, p=0.385 . * * \chi^{2}=0.161, p=0.688$.

Abbreviations: dMMR, deficient DNA mismatch repair; MMR, DNA mismatch repair; pMMR, proficient DNA mismatch repair; TIL, tumor-invading lymphocyte.

\section{Discussion}

The MMR system is made up of $\mathrm{dMMR}$ and $\mathrm{pMMR}$. MLH1 and MSH2 are major proteins of MMR. Deficient MLH1 or/and MSH2 and MSI status are defined as dMRR. ${ }^{10-12}$ According to prior studies, lack of MSH2 or/and MLH1 is a common phenomenon in cervical intraepithelial neoplasia and cervical cancer. ${ }^{13,14}$ The MSI of cervical cancer was $6.1 \%$ in this study, which was consistent with the report by Ercoli et al, ${ }^{15}$ but was less than the $12.6 \%$ reported by Baay et al. ${ }^{16}$ We reason that the source of cases and single-nucleotiderepeat markers may lead to these variations. In the study, the ratio of dMMR was $25.8 \%$ and the rates of dMMR varied greatly when analyzed in subtypes with different childbearing history, abortion history, and tumor differentiation. Because more incidences of childbearing and abortion could result in higher dMMR rates, we therefore surmise that these two factors may play crucial effects in healthy women, especially in the endometrial carcinoma uterus, where higher rates of dMMR are exhibited. ${ }^{17,18}$ Notably, cervical cancer originates from uterus-like endometrial carcinoma, indicating there should be a certain proportion of $\mathrm{dMMR}$ in cervical cancer. In a previous study of colon cancer, dMMR was significantly correlated with the degree of cancer cell differentiation; ${ }^{19}$ the study also explored the same correlation in cervical cancer.

This research explored whether positive expression of PD-L1 in cancer cells and TILs and of PD-1 in TILs was a common phenomenon in cervical cancer (positive rates were $59.1 \%, 47.0 \%$, and 60.6\%). For PD-L1 in cancer cells, more incidences of childbearing and abortion enhanced expression. Meanwhile, abortion history also enhanced the expression of PD-1 in TILs and more incidences of childbearing enhanced the expression of PD-L1 in TILs. A previous report showed that the PD-1/PD-L1 pathway was closely related to pregnancy history, ${ }^{20}$ pregnancy could change the immune status of women, especially the microenvironment immune status of the uterus including the cervical environment, and negative immune regulation pathways including the PD-1/PD-L1 pathway could be activated when pregnant. Cervical cancer is a common oncology from the uterus, and more incidences of childbearing and abortion were also risk factors for cervical cancer, so it was supposed that the PD-1/PD-L1 negative immune pathway was closely related to cervical cancer, and more incidences of childbearing and abortion could lead to overexpression of PD-L1 and PD-1. Furthermore, the study found that women aged between 35 and 55 years with cervical cancer had the highest PD-L1 expression in TILs and women older than 55 years had the lowest PD-L1 expression in TILs. Some previous reports claimed that some elderly 
Table 4 General characteristics of 66 cases

\begin{tabular}{|c|c|c|c|c|c|c|c|c|c|}
\hline & \multicolumn{2}{|l|}{ MMR } & \multicolumn{3}{|c|}{ PD-LI in cancer cells } & \multicolumn{2}{|c|}{ PD-I in TILs } & \multicolumn{2}{|c|}{ PD-LI in TILs } \\
\hline & dMMR & pMMR & $\begin{array}{l}\text { Strong } \\
\text { positive }\end{array}$ & Positive & Negative & Positive & Negative & Positive & Negative \\
\hline \multicolumn{10}{|l|}{ Age } \\
\hline$<35$ years old & I & 4 & 0 & 3 & 2 & 3 & 2 & 2 & 3 \\
\hline $35-55$ years old & 3 & 21 & 7 & 9 & 8 & 13 & 11 & 20 & 4 \\
\hline$>55$ years old & 13 & 24 & 14 & 6 & 17 & 15 & 22 & 18 & 19 \\
\hline \multicolumn{10}{|l|}{ Childbearing history } \\
\hline $0-2$ times & 5 & 30 & 4 & 12 & 19 & 13 & 22 & 17 & 18 \\
\hline$\geq 3$ times & 12 & 19 & 17 & 6 & 8 & 18 & 13 & 23 & 8 \\
\hline \multicolumn{10}{|l|}{ Abortion history } \\
\hline Yes & 15 & 28 & 20 & 11 & 12 & 24 & 19 & 28 & 15 \\
\hline No & 2 & 21 & 1 & 7 & 15 & 7 & 16 & 12 & 11 \\
\hline \multicolumn{10}{|l|}{ Ethnicity } \\
\hline Uygur & II & 25 & 15 & 8 & 13 & 20 & 16 & 26 & 10 \\
\hline Han & 6 & 24 & 6 & 10 & 14 & 11 & 19 & 14 & 16 \\
\hline \multicolumn{10}{|l|}{ Differentiation } \\
\hline Low & 14 & 21 & 14 & 11 & 10 & 17 & 18 & 23 & 12 \\
\hline Middle & 3 & 28 & 7 & 7 & 17 & 14 & 17 & 17 & 14 \\
\hline
\end{tabular}

Abbreviations: dMMR, deficient DNA mismatch repair; MMR, DNA mismatch repair; pMMR, proficient DNA mismatch repair; TIL, tumor-invading lymphocyte.

cancer patients would find treatment with PD-1/PD-L1 antibody drugs useless, ${ }^{21,22}$ the lowest PD-L1 expression of TILs in older patients being a potential reason. According to the clinical characteristics, a better treatment effect should be achieved using PD-1/PD-L1 antibody drugs in women 35-55 years old with cervical cancer and more incidences of childbearing and abortion history.

Further analysis of PD-L1/PD-1 expression in dMMR and pMMR was also performed, and results showed that in cancer cells the dMMR status was implicated with higher PD-L1 expression, which is consistent with similar reports of endometrial carcinomas. ${ }^{23}$ Recent studies reported that four factors contributed to the upregulation of PD-L1 in tumor cells, such as: 1) activation of the EGFR, MAPK, or PI3KAkt signaling pathway and high expression of STAT3 and HIF-1 protein could increase the high expression of PD-L1; 2) CD274 (coding PD-L1 gene, 9p24.1) amplification; 3) Epstein-Barr (EB) virus induction (EB virus positive gastric cancer and nasopharyngeal carcinoma, No9p24.1 gene amplification, also could show the high expression of PD-L1); and 4) epigenetic mechanisms. ${ }^{24}$ Although the regulatory mechanism of dMMR promoting PD-L1 high expression in cervical cancer cells was still unclear but combined with the function of MMR, we proposed that dMMR regulated PD-L1 expression by PD-L1 gene promoter region demethylation and $3^{\prime}$ untranslated region ( $3^{\prime}$-UTR) disruption of the PD-L1 gene. The disruption of the PD-L1 3'-UTR, which included the deletion, inserts, translocation, and tandem repeat of the gene sequence, could lead to the PD-L1 gene abnormal amplification, lastly leading to the PD-L1 protein abnormal high expression. ${ }^{25} \mathrm{dMMR}$ could cause microsatellite instability, which often leads to tandem repeat of gene sequences, ${ }^{26}$ and some researchers reported that dMMR could lead to all kinds of abnormal changes in the 3'-UTR of the functional gene and then to change in gene expression. ${ }^{27}$ So it was speculated that when the MMR is in a dMMR condition, which also could lead to PD-L1 gene $3^{\prime}$-UTR abnormal changes, this influenced the high expression of PD-L1 protein. Methylation of the PD-L1 gene had been reported, and PD-L1 expression had negative correlation with PD-L1 gene methylation; inversely, demethylation of the PD-L1 gene could increase the expression of PD-L1 protein. ${ }^{28,29}$ Grin et al preliminarily proved that the dMMR status closely related to demethylation of the functional gene, ${ }^{30}$ so it was believed that dMMR of cancer cells could lead to demethylation of the PD-L1 gene in the promoter region, and then it should affect the PD-L1 high expression.

Lastly, the study exhibited that patients with pMMR status had good relationships between PD-L1 in cancer cells and PD-1 in TILs, and PD-L1 in TILs, but in patients with dMMR PD-L1 in cancer cells had no correlation with PD-1 in TILs or PD-L1 in TILs. Both PD-1 in TILs and PD-L1 in cancer cells and TILs together form the PD-1/PD-L1 pathway, and the balance of the pathway is an important immune escape mechanism of tumor cells; ${ }^{1}$ in pMMR status, the balance is normal so there is a good relation between the three indexes. 

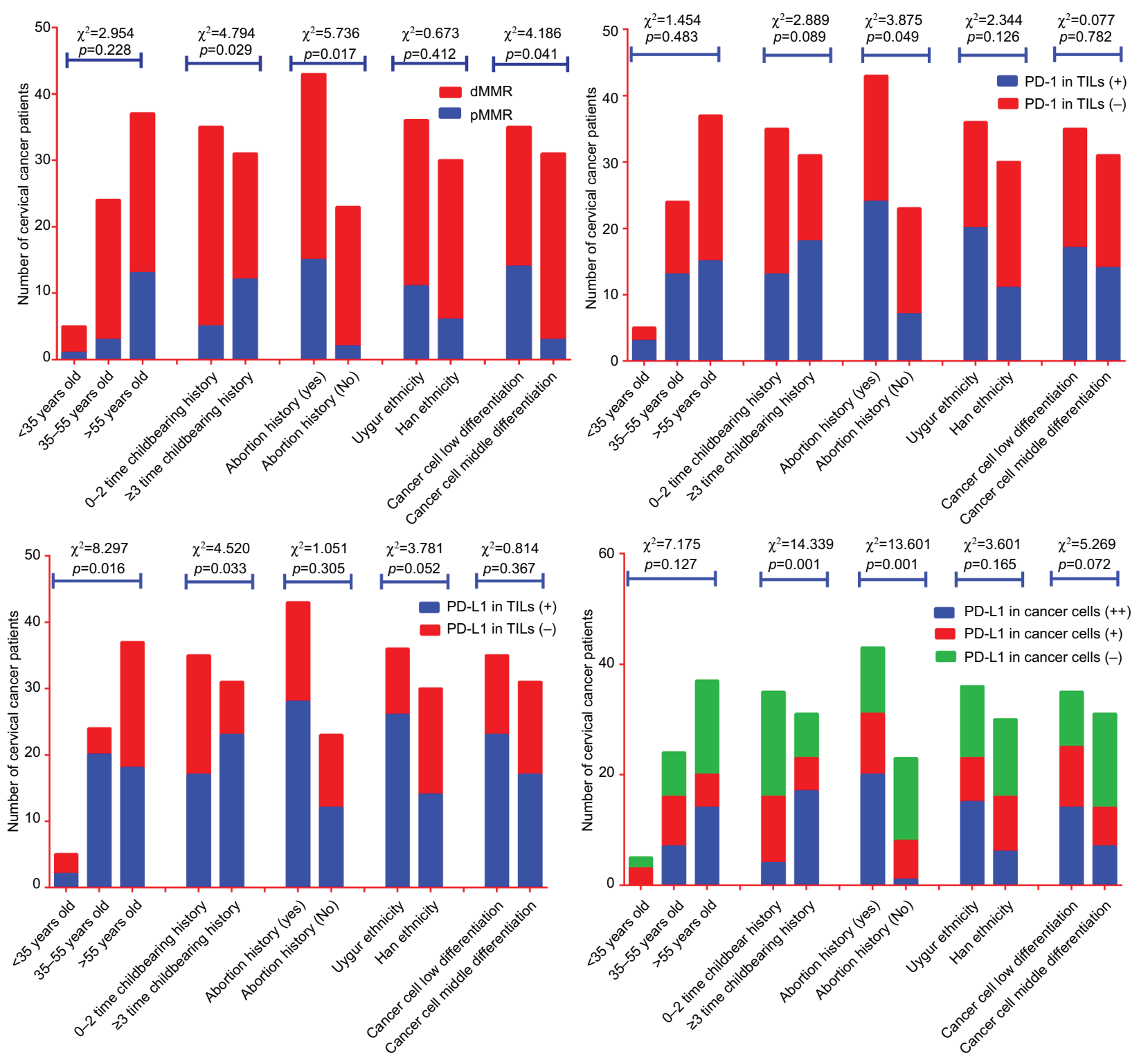

Figure 4 Clinical significance of mismatch repair and the PD-I/PD-LI pathway in 66 patients.

Abbreviations: $\mathrm{dMMR}$, deficient DNA mismatch repair; pMMR, proficient DNA mismatch repair; TIL, tumor-invading lymphocyte.

When patients are associated with dMMR status, high PD-L1 expression in cancer cells contributes to loss of balance, demonstrating no relationships between PD-L1 in cancer cells and PD-1/PD-L1 in TILs. Meanwhile, other immune molecules, such as immunocytokines, could help to repair the imbalance, and then the tumor microenvironment should form a precarious balance. PD-1/PD-L1 antibody drugs are capable of breaking the precarious balance, which subsequently achieves satisfactory therapeutic effects.

\section{Conclusion}

This study showed that dMMR status and PD-L1 expression in both cancer cells and TILs and PD-1 expression in TILs are common in cervical cancer patients. More incidences of childbearing and abortion act as the most important clinical characteristics for PD-1/PD-L1 expression in cervical cancer patients. Higher PD-L1 in cancer cells could be observed when patients are under dMMR status, rather than under pMMR status, suggesting that cervical cancer patients with dMMR may achieve higher therapeutic effects with PD-1/ PD-L1 antibody drugs. Admittedly, more tissue samples and further mechanisms should be added to future studies.

\section{Acknowledgment}

We thank Doctor Lu Xiao-xiong from Baylor College of Medicine for his assistance in English language copy editing. 


\section{Disclosure}

The authors report no other conflicts of interest in this work.

\section{References}

1. Villasboas JC, Ansell S. Checkpoint inhibition: programmed cell death 1 and programmed cell death 1 ligand inhibitors in Hodgkin lymphoma. Cancer J. 2016;22(1):17-22.

2. Frimer M, Levano KS, Rodriguez-Gabin A, et al. Germline mutations of the DNA repair pathways in uterine serous carcinoma. Gynecol Oncol. 2016;141(1):101-107.

3. Weber TK, Chin HM, Rodriguez-Bigas M, et al. Novel hMLH1 and hMSH2 germline mutations in African Americans with colorectal cancer. JAMA. 1999;281(24):2316-2320.

4. Le DT, Uram JN, Wang H, et al. PD-1 blockade in tumors with mismatch-repair deficiency. $N$ Engl J Med. 2015;372(26): 2509-2520.

5. Lin AY, Lin E. Programmed death 1 blockade, an Achilles heel for MMR-deficient tumors? J Hematol Oncol. 2015;8:124.

6. Le DT, Uran JN, Wang H, et al. PD-1 blockade in tumors with mismatchrepair deficiency. $N$ Engl J Med. 2015;375:2509-2520.

7. Lee V, Le DT. Efficacy of PD-1 blockade in tumors with MMR deficiency. Immunotherapy. 2016;8(1):1-3.

8. Lee SJ, Yang A, Wu TC, Hung C-F. Immunotherapy for human papillomavirus-associated disease and cervical cancer: review of clinical and translational research. J Gynecol Oncol. 2016;27(5):e51.

9. Dijkstra KK, Voabil P, Schumacher TN, Voest EE. Genomics- and transcriptomics-based patient selection for cancer treatment with immune checkpoint inhibitors: a review. JAMA Oncol. 2016;2(11):1490-1495.

10. Kim JE, Hong YS, Ryu MH, et al. Association between deficient mismatch repair system and efficacy to irinotecan-containing chemotherapy in metastatic colon cancer. Cancer Sci. 2011;102(9):1706-1711.

11. Kwasniewska A, Postawski K, Gozdzicka-Józefiak A, Zdunek M, Korobowicz E, Miturski R. Immunohistochemical detection of hMLH1 and hMSH2 proteins in vulvar carcinoma. Int $J$ Mol Med. 2005;15(6):955-961.

12. Castrilli G, Fabiano A, La Torre G, et al. Expression of hMSH2 and hMLH1 proteins of the human DNA mismatch repair system in salivary gland tumors. J Oral Pathol Med. 2002;31(4):234-238.

13. Nijhuis ER, Nijman HW, Oien KA, et al. Loss of MSH2 protein expression is a risk factor in early stage cervical cancer. J Clin Pathol. 2007;60(7):824-830

14. Ciavattini A, Piccioni M, Tranquilli AL, Filosa A, Pieramici T, Goteri G. Immunohistochemical expression of DNA mismatch repair (MMR) system proteins (hMLH1, hMSH2) in cervical preinvasive and invasive lesions. Pathol Res Pract. 2005;201(1):21-25.
15. Ercoli A, Ferrandina G, Genuardi M, et al. Microsatellite instability is not related to response to cisplatin-based chemotherapy in cervical cancer. Int J Gynecol Cancer. 2005;15(2):308-311.

16. Baay MF, Nakonieczny M, Wozniak I, et al. Microsatellite instability and HPV genotype in Polish women with cervical cancer. Eur J Gynaecol Oncol. 2009;30(2):162-166.

17. Köbel M, Tessier-Cloutier B, Leo J, et al. Frequent mismatch repair protein deficiency in mixed endometrioid and clear cell carcinoma of the endometrium. Int J Gynecol Pathol. 2017;36(6):555-561.

18. Rubio I, Ibáñez-Feijoo E, Andrés L, et al. Analysis of Lynch syndrome mismatch repair genes in women with endometrial cancer. Oncology. 2016;91(3):171-176.

19. Wang JB, Ma DL, Li JY, Sun QD, Liu YE. Association between expression of DNA mismatch repair genes and clinical features and prognosis of patients with radical resection of colon cancer. Genet Mol Res. 2016;15(3).

20. Li G, Lu C, Gao J, et al. Association between PD-1/PD-L1 and T regulate cells in early recurrent miscarriage. Int J Clin Exp Pathol. 2015;8(6): 6512-6518.

21. Brower V. Hyperprogressive disease with anti-PD-1 and anti-PD-L1. Lancet Oncol. 2016;12:e527.

22. Champiat S, Dercle L, Ammari S, et al. Hyperprogressive disease is a new pattern of progression in cancer patients treated by anti-PD-1/ PD-L1. Clin Cancer Res. 2017;23(8):1920-1928.

23. Sloan EA, Ring KL, Willis BC, Modesitt SC, Mills AM. PD-L1 expression in mismatch repair-deficient endometrial carcinomas, including Lynch syndrome-associated and MLH1 promoter hypermethylated tumors. Am J Surg Pathol. 2017;41(3):326-333.

24. Boussiotis VA. Molecular and biochemical aspects of the PD-1 checkpoint pathway. N Engl J Med. 2016;375(18):1767-1778.

25. Kataoka K, Shiraishi Y, Takeda Y, et al. Aberrant PD-L1 expression through $3^{\prime}$-UTR disruption in multiple cancers. Nature. 2016;534(7607):402-406.

26. Li YC, Korol AB, Fahima T, Nevo E. Microsatellites within genes: structure, function, and evolution. Mol Biol Evol. 2004;21(6):991-1007.

27. Gevensleben H, Holmes EE, Goltz D, et al. PD-L1 promoter methylation is a prognostic biomarker for biochemical recurrence-free survival in prostate cancer patients following radical prostatectomy. Oncotarget. 2016;7(48):79943-79955.

28. Goltz D, Gevensleben H, Dietrich J, Dietrich D. PD-L1 (CD274) promoter methylation predicts survival in colorectal cancer patients. Oncoimmunology. 2016;6(1):e1257454.

29. Kishore S, Piscuoglio S, Kovac MB, et al. 3'-UTR poly(T/U) tract deletions and altered expression of EWSR1 are a hallmark of mismatch repair-deficient cancers. Cancer Res. 2014;74(1):224-234.

30. Grin I, Ishchenko AA. An interplay of the base excision repair and mismatch repair pathways in active DNA demethylation. Nucleic Acids Res. 2016;44(8):3713-3727.
Cancer Management and Research

\section{Publish your work in this journal}

Cancer Management and Research is an international, peer-reviewed open access journal focusing on cancer research and the optimal use of preventative and integrated treatment interventions to achieve improved outcomes, enhanced survival and quality of life for the cancer patient The manuscript management system is completely online and includes

\section{Dovepress}

a very quick and fair peer-review system, which is all easy to use. Visit http://www.dovepress.com/testimonials.php to read real quotes from published authors. 